\title{
General Discussion to papers of Dr Hernandez et al., Dr Alvin Freehafer, Dr S. M. Rezaian et al. and Dr Chantraine
}

DR GRIFFITHs (Australia). From the last paper, I noted that on two of the three $\mathrm{X}$-rays we saw there was in fact quite marked para-articular ossification. If they had in fact this degree of para-articular ossification did they also have decubitus ulcers? We know we get hyperanaemia around infected areas. Is this a factor in causing osteoporosis and was it present in the cases that were discussed?

DR FORNER (Spain). Well these patients that I was showing had para-articular osteopathy but none of them had paraosteopathy (POA). We have a patient who had an increase of the femur and he didn't have any POA. We have shown then that osteoporosis and POA could sometimes appear following decubitus ulcer or infection but also before.

Dr HAChEN (Switzerland). Just a short comment on Forner's paper. He said he had no more incidence than 20 per cent of para-articular ossification, and he said there was no age correlation. He had a group between zero and 20 years of age. If he had broken it down into two groups 0 to IO and Io to 20 , he would most likely have seen that there was zero per cent of POA in children below the age of Io. There is a correlation below the age of let's say from Io to 20 where there must be a significant low incidence of POA.

DR ForNer. That is absolutely true. Until then we didn't have any para-articular ossification, so probably all of them are obviously from the age of ro upwards.

DR Michaelis (G.B.). Dr Chantraine has obviously a very sophisticated outfit for biochemical examination in osteoporosis. I have for many years tried to find somebody who will continue work done in Paris exactly 60 years ago about the content of fluorides in bone, which has not been repeated in the proper way since, and which seems to me to have a definite bearing on osteoporosis of varying kinds. According to the French authors of the time-I have forgotten their names-the fluorine increases as bone ages, but it is higher in the brittle bones of old people. It would be very interesting to see whether the osteoporosis in paraplegia that is below the level of the lesion only has anything in common with that particular type of osteoporosis. In any case, I believe that the search for fluorine which is technically not easy would be a valuable addition to your biochemical research. 